\title{
La investigación participativa en niños como herramienta en la promoción de la salud para la prevención de la Enfermedad de Chagas en Yucatán, México
}

\author{
Angel G. Polanco-Rodríguez, Hugo A. Ruiz-Piña, Fernando I. Puerto \\ Centro de Investigaciones Regionales "Dr. Hideyo Noguchi", Universidad Autónoma de Yucatán
}

\section{RESUMEN}

Introducción. La Investigación Participativa (IP), es una herramienta que puede abordarse para el análisis y mejoramiento de procesos socioculturales, medioambientales, y de salud pública. La participación de niños en edad escolar en la apropiación del conocimiento sobre la Enfermedad de Chagas (EC), puede romper el ciclo de transmisión. La EC es un problema de salud pública que afecta principalmente a comunidades rurales endémicas de países en desarrollo.

Objetivo: Desarrollar una estrategia para la adquisición y transferencia de nuevos conocimientos en niños y niñas, debido a la falta de información acerca del ciclo de transmisión de la EC .

Material y Métodos: Estudio socioambiental, realizado con un grupo de 48 niños y niñas de una comunidad en pobreza extrema en Yucatán, México. Mediante herramientas de IP y con el apoyo de las familias, autoridades ejidales y escolares, se impartieron talleres educativos a niños y niñas de educación básica para conocer el ciclo de transmisión de la EC y ayudar a prevenirla, debido a la abundancia del vector en el área de estudio. Se implementó un programa denominado "Pequeños Investigadores", para iniciar procesos de apropiación y socialización del conocimiento en la comunidad.

Resultados: El nuevo conocimiento adquirido por el grupo de niñas y niños fue aprovechado y compartido a sus familias, compañeros de escuela, y círculos sociales, fomentando la prevención de la enfermedad. Fueron recolectados 182 triatomas a nivel domiciliar y peri domiciliar.

Conclusiones: Los nuevos conocimientos, actitudes y prácticas adquiridos por los niños y las niñas bajo esquemas de participación, resulta benéfico para la prevención de la EC. Se recomienda al sector oficial la participación en la promoción de la salud en niños y niñas bajo el esquema del presente estudio en países en desarrollo.

Palabras clave: Enfermedad de Chagas, niños, niñas, promoción de la salud, investigación participativa

\footnotetext{
Autor para correspondencia: Angel G. Polanco-Rodríguez, Centro de Investigaciones Regionales "Dr. Hideyo Noguchi", Avenida Itzaés No. 490 x 59, CP 97000, Mérida, México E-mail: polanco07@gmail.com
}

Recibido: el 04 de abril de 2017 Aceptado para publicación: el 21 de agosto de 2017 Publicado: el 1 septiembre de 2017

Copyright (C) 2017 por autores (s) y Revista Biomédica.

Este trabajo esta licenciado bajo las atribuciones de la Creative Commons (CC BY).

http://creativecommons.org/licenses/by/4.0/

Este documento está disponible en https://doi.org/10.32776/revbiomed.v28i3.573 
Polanco-Rodríguez, et al

\begin{abstract}
Participatory research on child's as a tool in the health promotion for the prevention of chagas disease in Yucatán, México

Introduction. Participatory Research (PR) is a tool that can be approached for the analysis of sociocultural, environmental, and public health processes. The participation of schoolage childrens in the appropriation of knowledge for the prevention of Chagas' Disease (CD) can break the cycle of transmission. CD is a public health problem that mainly affects rural endemic communities of developing countries.
\end{abstract}

Objective. To develop a strategy for the acquisition and transfer of new knowledge in childs, due to the lack of information about the transmission cycle of the EC.

Materials and Methods. A cross-sectional study was carried out with a group of 48 childrens from a community in extreme poverty in Yucatan, Mexico. Through PR tools and with the support of parents, ejidal and school authorities, educational workshops were given to children of the basic education to know the cycle of transmission, and to prevent $\mathrm{CD}$ due to the abundance of the vector in the study area. A program called "Small Investigators" was implemented to initiate processes of appropriation and socialization of knowledge in the community.

Results. The new knowledge acquired by the group of children was used and disseminated to their families, partners, and social circles, promoting the prevention of the disease. 182 triatomas were collected at home and peri domicile.

Conclusions. The new knowledge, attitudes and practices acquired by children under participation schemes is beneficial for the prevention of the Chagas Disease. The official sector is recommended to design the promotion health programs in children under the scheme of the present study in developing countries.

Key words: Chagas disease, childs, health promotion, participatory research

\section{INTRODUCCIÓN}

Actualmente, a nivel global, los problemas de salud, sociales y medioambientales, no deben abordarse por separado, ya que existen bajo un sistema integrado de interacciones que deben analizarse y adaptarse en un mundo complejo de rápidas transformaciones (1). Lo anterior impacta a las sociedades rurales, quienes continúan bajo un sistema complejo por resolver, padecen de pobreza, bajos niveles educativos, falta de saneamiento y recursos naturales degradados, requiriéndose de nuevos esquemas para lograr cambios para la prevención de enfermedades infecciosas. La EC, es reconocida por la Pan American Health Organization (PAHO), como un problema de salud pública en Latinoamérica, pues el tratamiento presenta diversas dificultades, y una eficacia limitada, ya que aún no existe vacuna para prevenirla. Hasta el momento, ninguna estrategia para su control o eliminación ha sido eficiente. En Latinoamérica, se calcula la existencia de 8 millones de infectados y una población en riesgo de 108.5 millones (2).

En México, se han realizado estudios epidemiológicos sobre esta enfermedad, con estimaciones para el año 2006, de 1'100,000 personas infectadas y una población en riesgo viviendo en áreas endémicas de 29'500,000 (3). El insecto vector, Triatoma dimidiata ( $T$. dimidiata), es una de las principales especies que transmiten al parásito Trypanosoma cruzi ( $T$. cruzi), agente causal de la EC en Centroamérica y en el sur de México. El ciclo doméstico de transmisión es, principalmente, en viviendas rurales o zonas marginadas urbanas, construidas con paredes de bajareque, adobe, techos de guano y lámina de cartón. Entre los principales reservorios domésticos se encuentran perros y gatos $\mathrm{y}$, entre los reservorios silvestres, los marsupiales del género Didelphis, juegan un papel epidemiológico crucial en la transmisión de T. cruzi debido a que se han adaptado a ambientes rurales y urbanos. A nivel domiciliar, los insectos 
vectores viven y se reproducen en grietas de las paredes y techos, en los muebles de las viviendas, en cuadros y en los espacios del traspatio como son, gallineros, contenedores de leña y la basura acumulada. En Yucatán, la EC, ha sido una de las más estudiadas, principalmente, la epidemiología, el vector, los reservorios, y los factores de riesgo asociados a la transmisión. Sin embargo, es recomendable que se aborden metodologías de participación comunitaria para la prevención de las enfermedades. Los resultados destacan la frecuencia y estacionalidad de $T$. dimidiata, principal vector en la Península de Yucatán, en el domicilio y peridomicilio de localidades rurales y urbanas. El control vectorial es una estrategia de las más eficaces para la prevención de la enfermedad, sin embargo, es eficiente, únicamente, para especies que presentan domiciliación, pero, en especies no domiciliadas, el control o la prevención de infestación se convierte en una estrategia poco útil debido a la reinfestación periódica o estacional de las casas para T. dimidiata (4-11).

La participación comunitaria, es una estrategia que promueve alianzas entre investigadores $\mathrm{y}$ actores sociales. El grupo investigador $\mathrm{y}$ los participantes, se involucran en un proceso colaborativo con base en las necesidades para la identificación y la reflexión de los problemas comunitarios, combinando el conocimiento de los investigadores con el del grupo participante. Esta interrelación, permitirá generar propuestas que se traduzcan en acciones de intervención para la generación de capacidades y promoción de la salud. Las experiencias aportadas en este proceso, resultan positivas por parte de la comunidad, como la organización de los actores sociales y los cambios de actitud con base en los intereses detectados. Por lo tanto, podríamos entender a la investigación basada en la participación comunitaria, como un proceso metodológico cualitativo que conjuga los saberes socioculturales con los conocimientos del grupo investigador para mejorar las condiciones de vida de las comunidades. De este modo, el objetivo de la participación comunitaria es iniciar, desde una reflexión de la práctica educativa, la colaboración comunitaria, incluyendo el análisis de sus problemas, en donde los saberes de ambos grupos se complementen para transformarse en sujetos centrales del proceso, con un crecimiento horizontal mutuo, bajo una estructura didáctica flexible y disponible para el cambio (9-19).

El objetivo del estudio, fue desarrollar una estrategia para la adquisición de conocimientos en niños y niñas de educación básica, acerca del ciclo de transmisión y prevención de la EC, en una comunidad maya en pobreza extrema, con el apoyo y participación tanto de los padres de familia como de los docentes y autoridades ejidales.

\section{MATERIALES Y MÉTODOS.}

Este estudio socio ambiental, incluyó la utilización de herramientas de investigación participativa y participación comunitaria, en el cual se desarrollaron talleres con la asistencia de niñas y niños para evaluar la adquisición de conocimientos sobre el ciclo de transmisión de la $\mathrm{EC}$; estos talleres contaron con la participación con 48 estudiantes de tercero a sexto grado de la Escuela Primaria "Cristóbal Colón", de la localidad de San Pedro Chacabal en el municipio de Motúl, Yucatán, México.

La comunidad de San Pedro Chacabal, Motúl, México, Yucatán, está en las coordenadas $21^{\circ}$ $07^{\prime} 50^{\prime}$ ' N, 89 11' 56" O, (Figura 1); el mapa de paisajes de Yucatán indica que este ejido se encuentra ubicado en la unidad de paisaje de la planicie de Telchac Pueblo y forma parte de la denominada plataforma yucateca, con suelos, predominantemente, pedregosos con potencial agrícola bajo. Tiene una extensión territorial de 178 ha, distribuída de la siguiente manera: dos áreas parceladas, una al Norte de 62 ha, una al Sur de 107 ha; una parcela escolar de 4 ha., y un área de asentamientos humanos de 5 ha. Es 
Polanco-Rodríguez, et al

una comunidad maya pequeña en cuanto a traza urbana. La información poblacional indica que la localidad tiene 658 habitantes, de los cuáles 327 son mujeres y 331 son hombres; cuentan con un grado escolar promedio de 3.18 , su población económicamente activa es de 199 y la población económicamente inactiva es de 229 (20). talleres comunitarios sobre prevención de la EC, conservación del medio ambiente y del agua, prácticas de reforestación con la participación de los jóvenes, diseño y elaboración de un Programa de Ordenamiento Ecológico Territorial a escala ejidal con agricultores y ancianos de la comunidad.

\section{Estado de Yucatán, Dvisión Municipal}

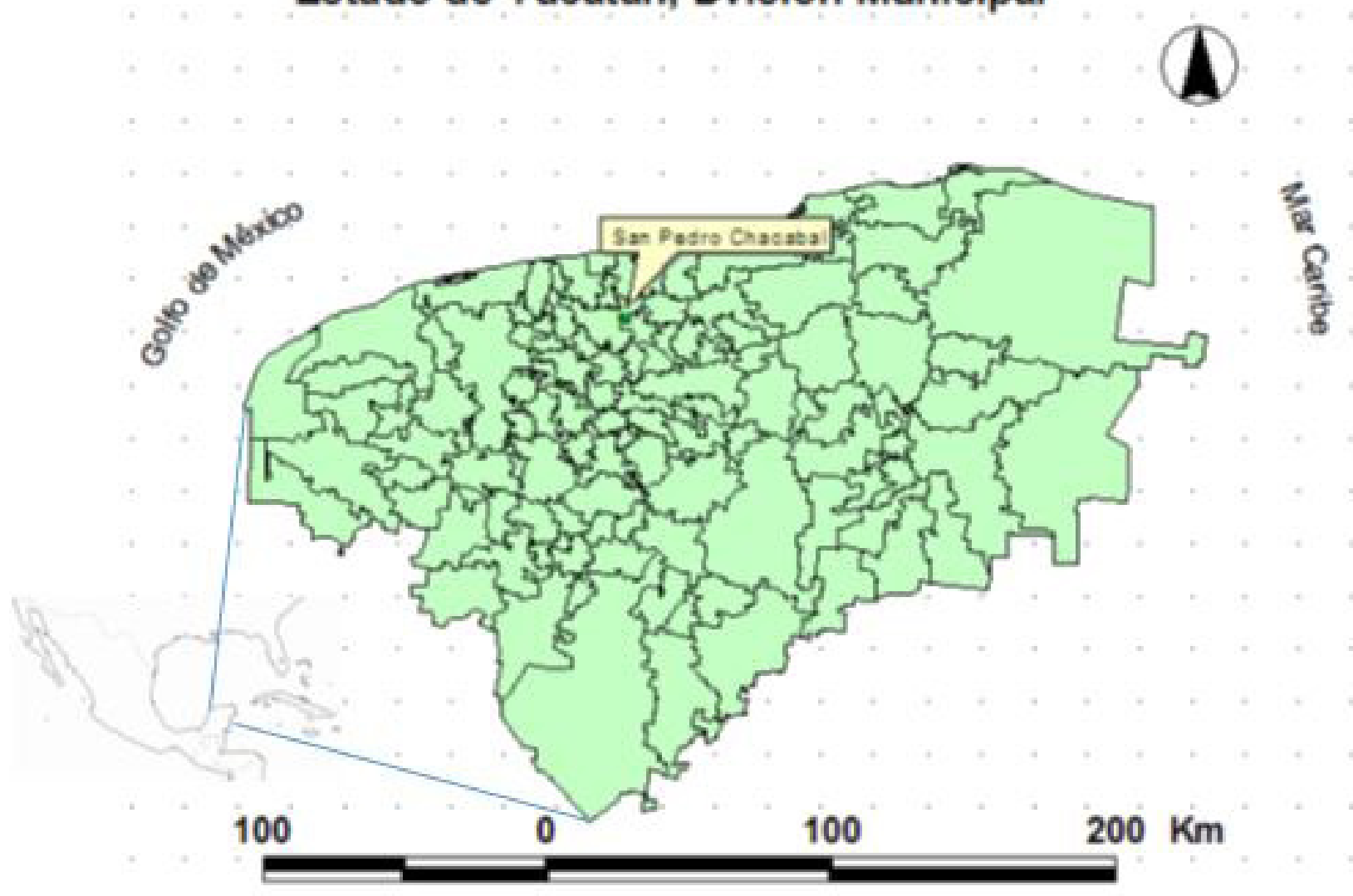

Figura. 1. Lugar de estudio de San Pedro Chacabal, Yucatán, México

El proyecto integral tuvo un enfoque ecosistémico para la generación de capacidades en 35 familias, basado en metodologías de investigación participativa. Se incluyeron proyectos productivos agroecológicos, pro-ducción de pequeñas especies, a nivel traspatio, para la generación de alimentos y alivio a la pobreza, producción de tilapia,
Para la organización de los talleres, se realizaron gestiones con autoridades educativas y ejidales; así mismo, se obtuvo el consentimiento informado de los padres y madres de familia, autoridade y de los niños y las niñas.

Una vez adquiridos los permisos correspondientes, se invitó a participar a los talleres 
comunitarios a estudiantes de la Escuela Primaria "Cristóbal Colón" de las 35 familias participantes en el proyecto integral. Posteriormente, se identificaron actores e informantes claves para el desarrollo del proyecto, involucrando autoridades municipales, docentes de la escuela, padres y madres de familia, niñas y niños.

En las sesiones de los talleres teórico-prácticos e inspección entomológica participaron 48 estudiantes de tercero a sexto grado de educación básica, con edades promedio de 8 a 12 años (28 niños y 20 niñas), 10 padres de familia, 10 madres de familia, dos autoridades ejidales, dos docentes, y la representante de los padres y madres de familia de la escuela, haciendo un total de 73 personas. Los talleres se realizaron en cuatro días en salones de la Escuela Primaria con duración de 24 horas, 16 horas teóricas y ocho prácticas (Cuadro 1).

Se diseñaron y usaron diversos materiales didácticos, como presentaciones digitales, dinámicas de juegos sobre prevención de la EC, cuentos acerca del ciclo de transmisión de la EC y concursos de armado de rompecabezas con figuras del vector, cuyo objetivo era reconocer e identificar a $T$. dimidiata y el ciclo de transmisión de la EC.

Con el propósito de conformar un diagnóstico inicial de conocimientos sobre la EC y el ciclo de transmisión en niños, se diseñó y aplicó una entrevista semiestructurada. Se obtuvo una muestra para colectar 120 entrevistas en el proyecto integral con el software estadístico Statistic. La estructura de la base de datos, consideró campos con registros digitales que incluyeron conocimientos sobre temas salud y de la EC, acerca del vector de la EC, ciclo de transmisión, riesgos del medio ambiente y reservorios, condiciones de vivienda, producción en el traspatio, animales domésticos.

La información fue procesada con el paquete SPSS (Statistical Packet for Social Sciences).

Se administró, además, un instrumento para evaluar el conocimiento adquirido al final del estudio sobre la EC y su ciclo de transmisión, en el que se incluyeron indicadores sobre conocimientos y ciclo de transmisión de la enfermedad, actividades en el solar, cambios de actitud, autogestión, manejo seguro del vector, manejo de recursos naturales y riesgos peridomiciliarios, liderazgo, organización y observación directa. El instrumento fue codificado y analizó con el paquete SPSS para obtener datos estadísticos.

Con base en los resultados del nivel de conocimiento de las niñas y de los niños acerca de la EC y el ciclo de transmisión, fueron diseñados los talleres comunitarios.

El desarrollo de los cuatro -talleres incluyó:

\section{Presentaciones digitales}

a) Evaluación sobre la percepción de los niños respecto al grado de conocimiento de la EC

b) Introducción, medio ambiente, y la EC

c) Ciclo de transmisión de la EC

d) Conocimientos y riesgos sobre el vector

\section{Dinámicas grupales}

a) Proyección del "Cuento educativo sobre la EC"

b) Trabajo en equipos para armar rompecabezas del vector

c) Concurso de dibujo del vector Triatoma dimidiata

d) Reconocimiento morfológico de los estadíos del vector

e) Sesiones de preguntas y respuestas sobre el vector

f) 10 sesiones demostrativas para identificación y captura del vector de forma segura en las viviendas y área peri-domiciliaria con los niños y las niñas, y los padres y madres de familia

g) Formación de un grupo de "Pequeños Investigadores"

h) Evaluación de los talleres

Vol. 28, No. 3, septiembre-diciembre de 2017 
Polanco-Rodríguez, et al

Cuadro 1

Descripción de los talleres

\begin{tabular}{|c|c|c|c|c|}
\hline TALLER 1 & OBJETIVO & METODOLOGÍA & DINÁMICAS & RESULTADOS \\
\hline $\begin{array}{l}\text { Ocho horas } \\
\text { en dos } \\
\text { sesiones }\end{array}$ & $\begin{array}{l}\text { Proceso de } \\
\text { sensibilización } \\
\text { a los niños } \\
\text { y las niñas } \\
\text { participantes. }\end{array}$ & $\begin{array}{l}\text { Presentación } \\
\text { digital: } \\
\text { Introducción a la } \\
\text { Enfermedad de } \\
\text { Chagas. } \\
\text { Descripción } \\
\text { de riesgos a la } \\
\text { Enfermedad de } \\
\text { Chagas. }\end{array}$ & $\begin{array}{l}\text { 1) Se proyectó } \\
\text { el "Cuento } \\
\text { educativo sobre } \\
\text { la Enfermedad de } \\
\text { Chagas" } \\
\text { 2) En equipos } \\
\text { armaron } \\
\text { rompecabezas del } \\
\text { vector T. dimidiata } \\
\text { 3) Concurso de } \\
\text { dibujo del vector } \\
\text { en el pizarrón } \\
\text { (niños y niñas). }\end{array}$ & $\begin{array}{l}\text { Se logró la participación e integración de } \\
\text { los niños y las niñas, así como de adultos } \\
\text { presentes en el taller. La adquisición } \\
\text { de nuevo conocimiento, analizando } \\
\text { información del grupo académico. Se } \\
\text { desarrolló un ambiente de confianza entre } \\
\text { el grupo técnico, entre las niñas y los niños, } \\
\text { y adultos participantes. Los niños y las } \\
\text { niñas conocían al vector, pero desconocían } \\
\text { la enfermedad que transmite y el ciclo de } \\
\text { transmisión de la Enfermedad de Chagas. } \\
\text { Es importante mencionar que los niños y } \\
\text { las niñas, así como los adultos participantes } \\
\text { en los talleres para la prevención de la } \\
\text { Enfermedad de Chagas, participaban } \\
\text { también en las actividades del proyecto } \\
\text { integral con sus familias y en la comunidad, } \\
\text { lo cual permitió una rápida integración para } \\
\text { el desarrollo de los talleres. }\end{array}$ \\
\hline TALLER 2 & OBJETIVO & METODOLOGÍA & DINÁMICAS & RESULTADOS \\
\hline $\begin{array}{l}\text { Ocho horas } \\
\text { en dos } \\
\text { sesiones }\end{array}$ & $\begin{array}{l}\text { Conocer } \\
\text { el ciclo de } \\
\text { transmisión de } \\
\text { la Enfermedad } \\
\text { de Chagas. }\end{array}$ & $\begin{array}{l}\text { Presentación } \\
\text { digital: } \\
\text { Ciclo de } \\
\text { Transmisión. }\end{array}$ & $\begin{array}{l}\text { 1) Dinámica } \\
\text { de tarjetas con } \\
\text { esquemas de } \\
\text { los estadíos del } \\
\text { vector para el } \\
\text { reconocimiento } \\
\text { morfológico. } \\
\text { 2) Sesión de } \\
\text { preguntas y } \\
\text { respuestas. }\end{array}$ & $\begin{array}{l}\text { Los niños y las niñas conocieron las etapas } \\
\text { morfológicas o estadíos del vector, el ciclo } \\
\text { de transmisión, así como información } \\
\text { sobre la enfermedad, los reservorios } \\
\text { mas importantes en las viviendas, } \\
\text { peridomicilio, y a nivel selvático. }\end{array}$ \\
\hline TALLER 3 & OBJETIVO & METODOLOGÍA & DINÁMICAS & RESULTADOS \\
\hline $\begin{array}{l}\text { Cuatro } \\
\text { horas }\end{array}$ & $\begin{array}{l}\text { Entrenamiento } \\
\text { para captura del } \\
\text { vector a nivel } \\
\text { domiciliar y } \\
\text { peri-domiciliar } \\
\text { con el apoyo de } \\
\text { los padres. }\end{array}$ & $\begin{array}{l}\text { Presentación } \\
\text { digital: } \\
\text { Entrenamiento } \\
\text { para la captura } \\
\text { segura del vector, } \\
\text { usando trampas, } \\
\text { guantes, pinzas, } \\
\text { frascos. }\end{array}$ & $\begin{array}{l}\text { Se seleccionaron } \\
\text { viviendas } \\
\text { para capturas } \\
\text { demostrativas. } \\
\text { Se capacitó a las } \\
\text { niñas y los niños } \\
\text { y adultos para la } \\
\text { captura del vector a } \\
\text { nivel domiciliario, } \\
\text { peri-domiciliario, y } \\
\text { selvático. }\end{array}$ & $\begin{array}{l}\text { Los niños y las niñas presenciaron las } \\
\text { prácticas demostrativas de captura del } \\
\text { vector a cargo del grupo investigador, } \\
\text { con participación de sus padres y madres, } \\
\text { reconociendo ninfas a nivel domiciliario. } \\
\text { Los niños y las niñas contribuyeron de } \\
\text { forma crucial al conocer sus propios } \\
\text { solares, peridomicilio, identificando los } \\
\text { vectores, así como a nivel vivienda. Algunos } \\
\text { niños que acompañaban a la milpa a sus } \\
\text { padres, lograron capturar triatomas a nivel } \\
\text { selvático. }\end{array}$ \\
\hline TALLER 4 & OBJETIVO & METODOLOGÍA & DINÁMICAS & RESULTADOS \\
\hline $\begin{array}{l}\text { Cuatro } \\
\text { horas }\end{array}$ & $\begin{array}{l}\text { Prácticas para } \\
\text { captura del } \\
\text { vector, aseo/ } \\
\text { mejoramiento } \\
\text { de la vivienda. }\end{array}$ & $\begin{array}{l}\text { Entrenamiento } \\
\text { para captura } \\
\text { del vector } \\
\text { como pequeños } \\
\text { investigadores. }\end{array}$ & $\begin{array}{l}\text { Captura del } \\
\text { vector en áreas } \\
\text { domiciliaria y } \\
\text { peridomiciliaria. } \\
\text { Mejora de las } \\
\text { condiciones de la } \\
\text { vivienda. }\end{array}$ & $\begin{array}{l}\text { Los niños y niñas, y los padres y } \\
\text { madres de familia tuvieron cambios } \\
\text { de actitud respecto a la problemática, } \\
\text { mostrando integración y organización. } \\
\text { Comprendieron y aplicaron las técnicas } \\
\text { de trampeo y colecta segura del vector. } \\
\text { Realizaron limpieza en las viviendas y } \\
\text { traspatio, en lo posible mejoraron sus } \\
\text { condiciones, colectando materiales donde } \\
\text { puede darse la colonización del vector. }\end{array}$ \\
\hline
\end{tabular}


i) Viajes de campo del grupo técnico cada dos días a la comunidad durante tres meses para retroalimentar el conocimiento en las niñas y los niños y recolectar los triatomas capturados.

\section{RESULTADOS}

El diagnóstico inicial para medir el conocimiento sobre la EC y su ciclo de transmisión indicó que el $100 \%$ de las niñas y de los niños, conocía al vector $T$. dimidiata con el nombre de "pic" y su forma de alimentación es con sangre, pero desconocían la enfermedad y el ciclo de transmisión. Creían normal encontrar al triatoma dentro y fuera de la vivienda, en los espacios peridomiciliarios. Se observó, por parte del grupo de niñas y niños, los padres y madres de familia, docentes y autoridades ejidales un desconocimiento generalizado, tanto acerca de la EC, como de su ciclo de transmisión, colonización, y los reservorios, domésticos y silvestres. Al finalizar los talleres teórico-prácticos, 95\% de las niñas y de los niños demostró haber adquirido nuevo conocimiento sobre el nombre de la enfermedad, nombre del vector, sitios de infestación, características de la enfermedad, ciclo de transmisión general y captura segura del vector con apoyo de sus madres y padres.

Por otra parte, el proceso fue evaluado por observación directa sobre los cambios de actitud respecto a los riesgos de la presencia del vector en las viviendas, traspatio y en el área peridomiciliar, observándose que con el nuevo conocimiento adquirido, las niñas y los niños mostraron actitud de altos cuidados sobre los lugares de infestación del vector al interior de la vivienda y el riesgo involucrado, así como las técnicas de colecta intra y peridomiciliar.

La información colectada, a través de las entrevistas, muestra que las familias tienen animales, como aves y cerdos, en su solar para autoconsumo y construyen sus corrales y gallineros, sin embargo, durante el día sacan a los animales a pastorear para que se alimenten libremente en el solar y en los alrededores de la vivienda, introduciéndose en ellas, situación que, junto con los animales domésticos como perros y gatos, facilita la alimentación de vectores intradomiciliares con sangre, asegurando la infestación de las viviendas.

Con base en un nivel de participación de 95\% en los talleres de los estudiantes de la escuela "Cristobal Colón", se demostró el funcionamiento de las trampas de captura, dejándolas instaladas en las casas, así como la utilización de frascos, guantes y pinzas para capturar y envasar de manera segura al vector con apoyo de los adultos. Durante los talleres y en las visitas domiciliarias se explicó la importancia de la EC, el ciclo de transmisión, el proceso de infestación, promoviendo los cuidados, el aseo y el mejoramiento de la vivienda para prevenir los riesgos de infestación y transmisión de la enfermedad.

Las condiciones de vivienda de la población son precarias pues se encontro que, $79.2 \%$ tiene pisos de tierra; $20.8 \%$, tiene paredes de piedra; $62.5 \%$ tiene techos de paja o cartón y, en $40 \%$ de la población, duermen cuatro o cinco personas en una habitación pequeña, con alto grado de hacinamiento y bajo condiciones de habitabilidad restringidas. El número de habitaciones totales en las viviendas de la comunidad fue de uno o dos. $90 \%$ de las niñas y los niños generalmente no usa calzado ni dentro de la vivienda ni cuando juegan con sus amigos en la calle. Se observó escaso aseo personal, realizando sus actividades cotidianas bajo este escenario. Al interior de la vivienda se capturaron seis triatomas en el estadio de "ninfas", lo cual indica que han colonizado las viviendas, alimentándose de sus habitantes y animales domésticos.

Se observó y discutió con las niñas y los niños los riesgos anteriormente mencionados (Cuadro 2).

$\mathrm{La}$ encuesta entomológica realizada por las niñas y los niños con el apoyo de los adultos fué de 182 insectos (17 silvestres, 24 peridomiciliarios y141 domiciliarios) colectados en tres meses. Se reportaron 18 viviendas infestadas, lo que representó $51 \%$ de las viviendas estudiadas. La 
Polanco-Rodríguez, et al

\begin{tabular}{lc}
\multicolumn{2}{c}{$\begin{array}{c}\text { Cuadro 2 } \\
\text { Riesgos en las viviendas } \\
\text { asociados a la EC }\end{array}$} \\
\hline \multicolumn{2}{c}{ Falta de aseo y orden } \\
\hline Desconocen los lugares de anidación \\
Tienen animales domésticos dentro de la & $86 \%$ \\
vivienda & $100 \%$ \\
Entran aves al interior de la vivienda & $90 \%$ \\
Crían animales de traspatio & $98 \%$ \\
Grietas en las paredes & $77 \%$ \\
\hline
\end{tabular}

zona este de los asentamientos humanos de la localidad, fue donde se capturó el mayor número de vectores, probablemente, porque es la más cercana a las milpas de maíz, lugar donde hay más alimento para los reservorios de los que se alimenta el vector (Figura 2).
El grupo de investigación realizó viajes a la comunidad cada dos días durante tres meses para colectar los triatomas capturados en las trampas y proporcionar retroalimentación de conocimientos. Por otra parte, otro grupo de investigación realizó capturas nocturnas en áreas selváticas adyacentes a la localidad (Figura 2 ), llegándose a capturar, en ocho trampeos nocturnos, 544 triatomas adultos, lo cual indica abundancia del vector en la periferia de las viviendas, con una tasa de infección para $T$. cruzi de $3.7 \%$ (21).

La evaluación de la participación comunitaria en el proyecto global integral, incluyó la colaboración de las niñas y de los niños, obteniendo excelentes resultados al medir los indicadores sobre la integración familiar en la

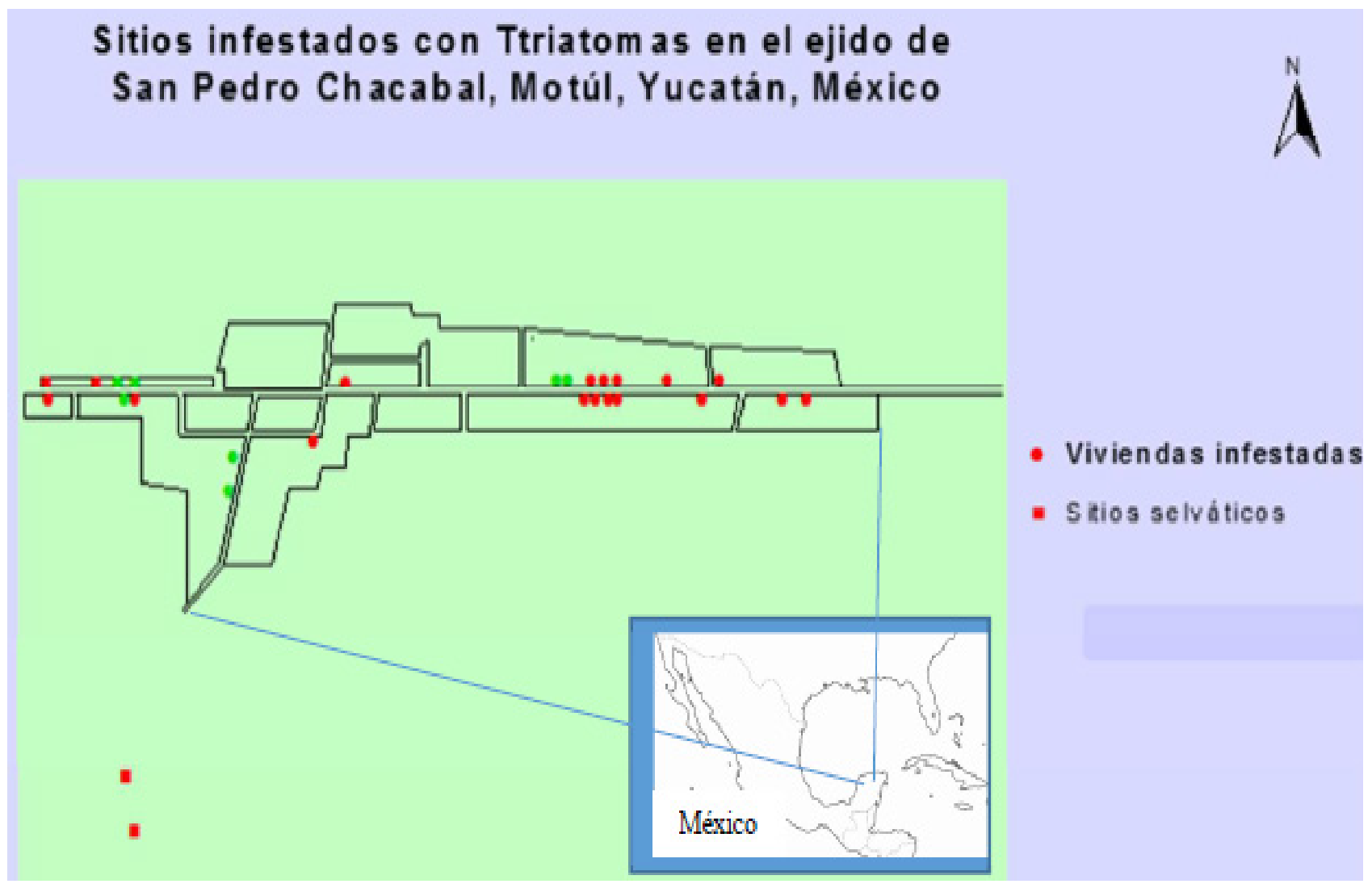

Figura. 2. Mapa de viviendas infestadas, San Pedro Chacabal, Yucatán, México 
participación de las actividades en el solar, así como en los cambios de actitud y adopción de nuevas prácticas para el cuidado de su salud en realción a la EC (Figura 3 ).
En la comunidad estudiada, tuvo lugar un proceso de apropiación y, posteriormente, extensión del conocimiento de las niñas y de los niños participantes en sus hogares para prevenir la EC.

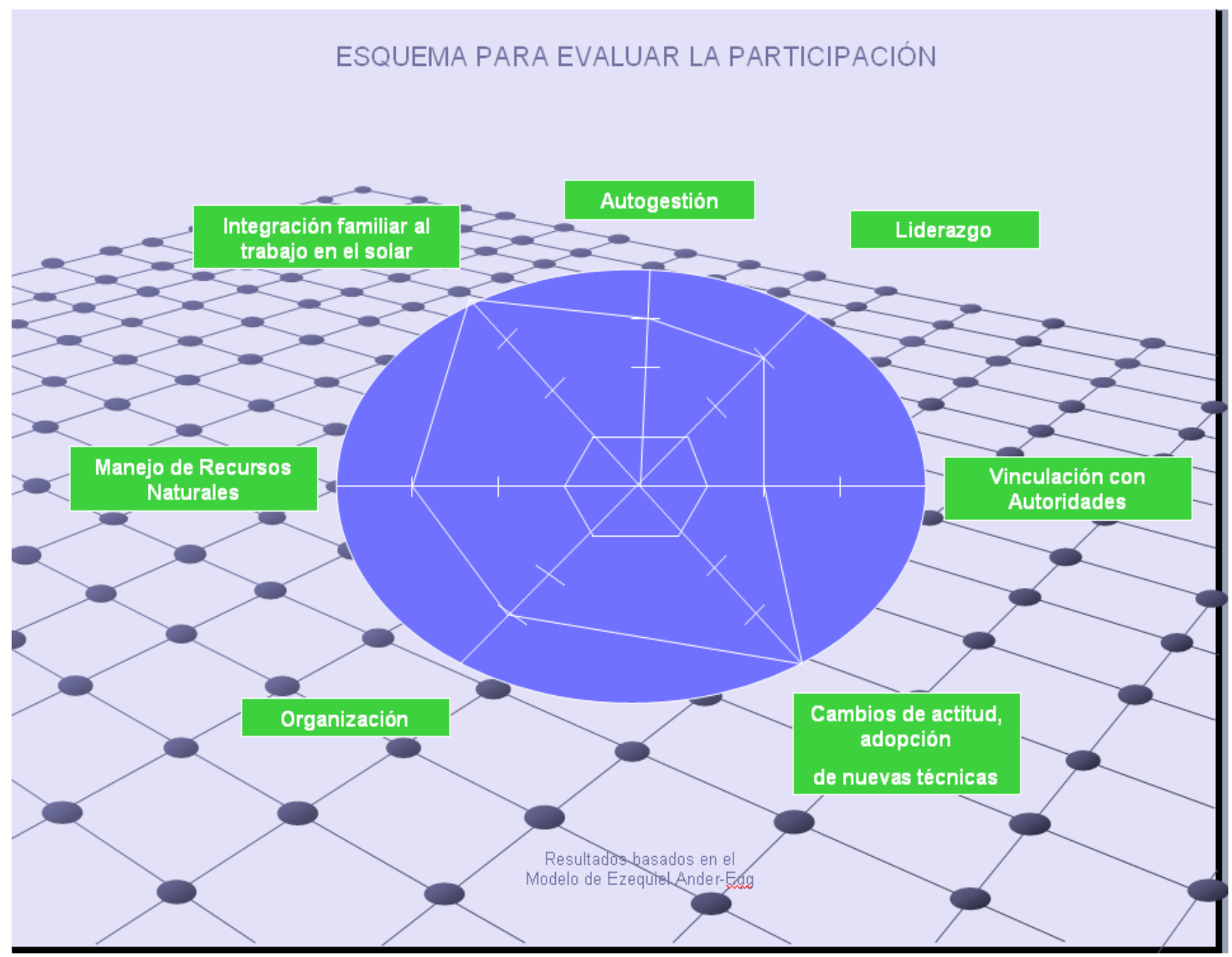

Figura. 3. Resultados basados en el Modelo de Ezequiel Ander Egg

\section{DISCUSIÓN}

Las comunidades rurales necesitan, tanto aplicar nuevas estrategias para romper el ciclo de transmisión de la EC, como la investigación participativa y enfoques multidisciplinarios socio ambientales, entendiendo que, su participación en procesos de adquisición de conocimientos sobre los riesgos ambientales e impactos en la salud, juegan un papel crucial para el control de la enfermedad (22-24).
Los padres y madres de familia apoyaron a sus hijas e hijos, al participar en las dinámicas y prácticas de captura del vector a nivel domiciliario y peridomiciliario, previo entrenamiento y suministro de trampas para captura del vector y materiales de seguridad. Así mismo, los docentes, una vez comprendida la información, lo compartieron en sus salones de clase, ampliándose el nuevo conocimiento en la comunidad. De esta forma, a través de los talleres y prácticas realizadas, se logró 
Polanco-Rodríguez, et al

informar y concientizar a las niñas y a los niños de la escuela primaria, a sus familias y a la comunidad en general. La investigación cualitativa, generalmente, no es considerada relevante en políticas educativas, sin embargo, deben ampliarse los objetivos para que sea incluída en el diseño de políticas públicas, para que genere nuevos conocimientos desde la base de grupos vulnerables en pobreza y pobreza extrema, lo que permitirá ir más allá de las políticas neoliberales de atención a la salud, y se promuevan estrategias de prevención (25-27).

Se han realizado estudios como en Comayagua, Honduras, de promoción para la salud en comunidades de intervención y comunidades control, obteniéndose mejores resultados en las comunidades de intervención, en donde las visitas domiciliarias para concientizar a la población acerca del vector y el mejoramiento de la vivienda tuvieron mejores resultados (28).

El hallazgo de ninfas indicó la colonización del vector en algunas viviendas, alimentándose de sus habitantes, animales domésticos y animales de traspatio. $100 \%$ de las viviendas visitadas tuvo, al menos, un perro, así como cría de aves de traspatio y, en algunos casos, cerdos (29).

En la región maya se requieren trabajos multidisciplinarios, que incluyan estudios de corte social participativo y de promoción de la salud ya que, generalmente, sólo están enfocados al área biomédica. Es recomendable analizar las condiciones sociales de pobreza y marginación, la falta de higiene y saneamiento, el mejoramiento de las viviendas, así como la convivencia con animales domésticos, ya que son factores de riesgo para la prevención de enfermedades (30).

La degradación ambiental juega un papel crucial en la dinámica poblacional del vector, así como las estrategias adaptativas en su migración hacia poblaciones humanas, para establecer su domiciliación. En la comunidad bajo estudio, la deforestación, temperatura, humedad, precipitación, cambios en el uso del suelo, alteración del hábitat, incendios forestales, pueden tener efectos en la movilidad del vector, infestando y colonizando viviendas en las poblaciones e incrementando la transmisión doméstica, tal como se reporta en un estudio en la Amazonia (31). Por otra parte, en la Península de Yucatán, debe tomarse en cuenta que es una zona geográficamente vulnerable a los huracanes, al calentamiento global, con una región semi árida al norte, ubicada en la región con clima transicional, con más sensibilidad a cambios que dependen de las condiciones mundiales (32).

La evaluación de los datos obtenidos indica que las niñas y los niños participantes mejoraron significativamente sus conocimientos al adquirir y retener la información proporcionada en los talleres comunitarios acerca de la EC y su ciclo de transmisión. $100 \%$ de los niños y las niñas aprendieron a reconocer el vector $T$. dimidiata, como transmisor de la EC, sus diversos estadíos, los lugares donde se puede encontrar en el domicilio y peridomicilio, así como los animales domésticos de los que se alimenta y el alto riesgo del ser humano de que sea infectado. Los niños y las niñas comprendieron la importancia del saneamiento en sus viviendas para disminuir la infestación domiciliaria del vector.

$100 \%$ de las niñas y los niños reconocieron fácilmente al reservorio Didelphis virginiana, principal reservorio de T. cruzi, conocido como zorro en Yucatán, pero desconocían que el vector se alimenta de ellos, así como de los animales domésticos como perros, gatos y animales de traspatio como aves y cerdos, propiciando un riesgo potencial de contraer la enfermedad para ellos y sus familias.

$100 \%$ de las niñas y los niños, practicaron ser "Pequeños Investigadores", al mostrar habilidades para la búsqueda del vector en la vivienda y peridomicilio, así como para la captura segura con las herramientas proporcionadas y con la ayuda de los adultos.

Los nuevos conocimientos, actitudes y prácticas adquiridos bajo esquemas de participación para 
la prevención de la EC, resultaron benéficos para la comunidad ya que, antes del proceso de adquisición del conocimiento, los niños y las niñas, no tenían información acerca de la EC, ni del vector ni de su ciclo de transmisión. El proceso participativo de los niños y las niñas genera expectativas, al haber adquirido información sobre el vector, su ciclo de transmisión, sus hábitos alimenticios, lugares para anidar en las viviendas y peridomicilio, así como los potenciales riesgos a la salud. Experiencias similares han sido realizadas por un grupo de voluntarios japoneses para el control de vectores de la EC en Guatemala, promoviendo el cuidado de la salud en escuelas primarias, con la participación de niñas y niños, maestros y maestras y con el apoyo de agencias de gobierno locales (33).

Por otra parte, la integración de factores Ecológicos-Biológicos-Sociales, bajo escenarios de globalización, debe analizarse en problemas de salud poblacional de manera multidisciplinaria. El cambio climático y la modificación del uso del suelo afectan la dinámica del vector, ocasionando que las poblaciones de $T$. dimidiata puedan estar desplazándose, en un intento por buscar nuevos ecotopos para su sobrevivencia, invadiendo asentamientos humanos, como es el caso de esta comunidad. Estos factores "EcoBio-Sociales" pueden influir en la transmisión de la enfermedad. La abundancia de triatomas en las áreas cercanas a la comunidad, así como las contingencias ambientales, pueden poner en alto riesgo la salud humana (34).

Un taller realizado por la Fundación Edna McConnell Clark en Kentucky, USA, 1994 (35), concluyó de manera importante, que los niños requieren conocimientos que los ayuden a resolver sus problemas cotidianos de salud, así como tener habilidades prácticas que les permitan reconocer y protegerse contra las enfermedades. Con base en lo anterior, las comunidades que posean mayor información sobre el problema, mostrarán una disminución significativa de los factores de riesgo.
Las condiciones de pobreza y enfermedades asociadas, continúan frenando el desarrollo de comunidades rurales mayas; el bajo nivel educativo de las familias, el deterioro del medio ambiente, ya sea por contingencias ambientales o por acciones antropogénicas, aunados a la pobre percepción de la comunidad respecto al desarrollo y conservación del medio ambiente, carencias sociales básicas, como condiciones precarias de la vivienda, falta de empleo, falta de programas oficiales preventivos sobre la EC, continúan manteniendo las condiciones de pobreza, pobreza extrema y, por lo tanto, un alto riesgo a enfermedades.

Esta experiencia preliminar puede ser un referente útil para estudios longitudinales, diseñando estrategias para medir la retención y transmisión del conocimiento en niñas y niños y sus familias, así como indicadores ambientales relacionados a la dinámica vectorial.

\section{REFERENCIAS}

1. Folke C, Carpenter S, Elmqvist T, Gunderson L, et al. Resilience and Sustainable Development: Building Adaptive Capacity in a World of Transformations. Hum Env. 2002 Aug; 31(1):437-40.

2. Panamerican Health Organization. Planes de Acción Integrados para la Prevención, Control y Eliminación de las Enfermedades Infecciosas Desatendidas. Lima: PAHO; [en línea] 2011 Octubre. [Fecha de acceso 3 Marzo 2017]. Disponible en: http://www.google. com.mx/url?sa $=\mathrm{t} \& \mathrm{rct}=\mathrm{j} \& \mathrm{q}=\&$ esrc $=\mathrm{s} \&$ source $=$ web $\& c d=2 \& v e d=0$ ahUKEwj7-tf3hsnPAhUF0oMKHc mLBAAQFgggMAE\&url=http\%3A\%2F\%2Fwww. paho.org\%2Fhq\%2Findex.php\%3Foption\%3Dcom docman\%26task\%3Ddoc_view\%26gid\%3D158 32\%26Itemid\%3D270\&usg=AFQjCNH_FvyL GD1tmd2rDNOeIcxc4G0H4Q\&sig2=6CrtEbvfjk4pIRFaQJUDw

3. Cruz-Reyes A \& Pickering-López JM. Chagas disease in Mexico: an analysis of geographical distribution during the past 76 years - A review. Mem. Inst. Oswaldo Cruz. 2006 Jun; 101 (4): 345-54.

4. Zeledón R Ponce C \& Mendez-Galvan J.F. 2007. Epidemiological, social and control determinants of Chagas disease in Central America and MexicoGroup discussion. Mem. Inst. Oswaldo Cruz. 2007 Jul; 102 (Suppl. 1); 45-46. 
Polanco-Rodríguez, et al

5. Zavala-Velázquez J. La enfermedad de Chagas en el Estado de Yucatán, México (1940-2002). Rev Biomed. 2003 Feb; 14(1):35-43.

6. Dumonteil E, Gourbiere S, Barrera-Pérez M, Rodríguez-Félix E, Ruíz-Piña H, Baños-López O. et al. Geographic distribution of Triatoma dimidiata and transmission dynamics of Trypanosoma cruzi in the Yucatan Peninsula of Mexico. American Trop Med Hyg J. 2002 Ago; 67 (2):176-83.

7. Dumonteil E \& Gourbiere S. Predicting Triatoma dimidiata abundance and infection rate: a risk map for natural transmission of Chagas disease in the Yucatan Peninsula of Mexico. Am J Trop Med Hyg. 2004 May; 70(5):514-19.

8. Dumonteil E, Ruíz-Piña $H$, Rodríguez-Félix E, Barrera-Pérez M, Ramírez-Sierra M, Rabinovich JE et al. Re-infestation of houses by Triatoma dimidiata after intra-domicile insecticide application in the Yucatan Peninsula, Mexico. Mem Instit Oswaldo Cruz. 2004 May; 99(3):253-6.

9. Dumonteil E, Ramírez-Sierra MJ, Ferral J, EuánGarcia M \& Chavez-Nuñez L. Usefulness of Community Participation for the Fine Temporal Monitoring of House Infestation by Non-Domiciliated Triatomines. Paras J. 2009 Apr; 95(2):469-71.

10. Mincler $M$ and Wallerstein N. Community Based Participatory Research for Health. New Jersey Wiley Inprint; 2008.

11. Ruiz-Piña H.A. y A. Cruz-Reyes. The Opossum Didelphis virginiana as a synanthropic reservoir of Trypanosoma cruzi in Dzidzilché, Yucatan, Mexico. Mem. Inst. Oswaldo Cruz. 2002 Jul; 97(5):613-20

12. Leonard A, Keys CB, Suarez-Balcazar Y, Taylor RR, Davis MI. Participatory community research: Theories and methods in action. Washington (DC): American Psychological Association; 2004.

13. Bergold J, \& Thomas S. Participatory Research Methods: A Methodological Approach in Motion. Forum: Qualitative Social Research. 2012 Jan; 13(1):1-31

14. Wallerstein N and Duran B. Community-Based Participatory Research Contributions to Intervention Research: The Intersection of Science and Practice to Improve Health Equity. Am J Public Health. 2010 Apr; 100(1):40-6

15. Dumas M J, Anderson G. Qualitative Research as Policy Knowledge: Framing Policy Problems and Transforming Education from the Ground Up. Education Policy Analysis Archives. 2014 Feb; 22(11):1-21.
16. Creswell, J.W. El Diseño de la Investigación Cualitativa. Adaptado de Research Design Qualitative \& Quantitative Approaches. Biblioteca Virtual, Universidad de Chile. [En línea] 2003 [Consultado 13 Marzo 2017] Disponible en URL:http://www. fcs.uner.edu.ar/libros/archivos/ebooks/Ciencia $\% 20$ \&\%20Tecnologia/InvestCualitativa.pdf

17. Ander-Egg E. Evaluación de Servicios y Programas Sociales. Siglo XXI de España. Fundación Dialnet. 175 p. ISBN: 84-232-0754-8. [En línea] 1992 [Consultado 17 Mayo 2017] Disponible en URL:https://www. researchgate.net/publication/31643199_Evaluacion de_servicios_y_programas_sociales_MJ_Aguilar_ Idanez_E_Ander-Egg

18. Freire P. Educación y Proyección Comunitaria. [En línea] 2011. [Consultado 8 de julio de 2016] Disponible en: URL: http://albilla-alba.blogspot. mx/2011/07/educacion-y-participacion-comunitaria. html

19. Freire P. Educación y Participación comunitaria. En: Política y Educación. Madrid: Siglo XXI, pp.7387. [En línea] 1993 [Consultado 13 Junio 2017] Disponible en URL: https://libros-gratis.com/ebooks/ politica-y-educacion-paulo-freire/

20. SEDESOL. Secretaría de Desarrollo Social. Catálogo de localidades. [En línea] 2005. [Consultado 8 Julio 2016] Disponible en: URL: http:// www.microrregiones.gob.mx/catloc/contenido. aspx?refnac $=310520017$

21. Rebollar-Téllez EA, Reyes-Villanueva F, EscobedoOrtegón J, Balám-Briceño $\mathrm{P}$, May-Concha $\mathrm{I}$. Abundance and Nightly Activity Behavior of a Sylvan Population of Triatoma dimidiata (Hemiptera: Reduviidae: Triatominae) from the Yucatan, México. Vec Ecol J. Dic 2009;34(2):304-10

22. Charron DF. Ecosystem Approaches to Health for a Global Sustainability Agenda. EcoHealth. Sep 2012;9(3):256-66. DOI: 10.1007/s10393-012-0791-5

23. Zeledón R \& Rojas JC. Environmental management for the control of Triatoma dimidiata (Latreille, 1811), (Hemiptera: Reduviidae) in Costa Rica: a pilot project. Mem Inst Oswaldo Cruz. Jun 2006;101(4):379-386.

24. Polanco-Rodríguez A.G. Alternativas sostenibles de desarrollo rural participativo para el mejoramiento de la calidad de vida en la comunidad Maya de San Pedro Chacabal, Motúl, Yucatán, México. Tesis para obtener el título de Maestría en Manejo y Conservación de Recursos Naturales Tropicales. Facultad de Veterinaria. Universidad Autónoma de Yucatán, México. 2007. 
Promoción de la salud en niños

25. Crocco L, Sanmartino M, Campaner G, Catalá S, Lobbia P, López A, Perasso L. et al. Chagas, Educación y Promoción de la Salud. Estrategias para abordar la problemática en diferentes contextos. Ed. SIMA, Córdoba, Argentina. 2011.

26. Hashimoto K, Zúniga C, Nakamura J, and Hanada K. Integrating an infectious disease programme into the primary health care service: a retrospective analysis of Chagas disease community-based surveillance in Honduras. BMC Health Services Research. 2015; 15:116. [En línea] 2015 [Consultado 17 Mayo 2017] Disponible en URL: https://bmchealthservres. biomedcentral.com/articles/10.1186/s12913-0150785-4

27. Cabrera Rufino MC, Suárez N, Infante C, Náquira C, García-Zapata MTA. Knowledge, attitudes, and practices concerning Chagas disease in schoolchildren from an endemic area in Peru. Cad. Saúde Pública. 2003 Feb;19(1):147-54

28. Avila Montes G, Martínez LM, Ponce C, Ponce E, Rosales R, Orellana LL, Quintana M. Participación Comunitaria para el Control de la Enfermedad de Chagas: Experiencia en una área Endémica de Honduras. Rev Med Hondur. 1996 Jun;64(2):52-9.

29. Gürtler RE, Wisniveski-Colli C, Solarz ND, Lauricella M, Bujas MA. Dynamics of transmission of Trypanosoma cruzi in a rural area of Argentina: II. Household infection patterns among children and dogs relative to the density of infected Triatoma infestans. Bull Pan Am Health Organ. 1987;21(:280-92.
30. Pacheco J, Lugo JA, Barrera MA, Tzuc L. Diagnóstico antropológico y médico sobre la Enfermedad de Chagas en poblaciones rurales de Yucatán. Ed. Universidad Autónoma de Yucatán-Conacyt; 2011.

31. Coimbra CEA. Human Settlements, Demographic Pattern, and Epidemiology in Lowland Amazonia: The Case of Chagas's Disease. Am Ant. 1988 Marzo;90(1):82-97. doi: 10.1525/ aa.1988.90.1.02a00060

32. Orellana R., Espadas C., Conde, C., Gay, C. Climate Change Scenarios in the Yucatan Peninsula to the year 2020. En: Libro de Memorias del Sixth Alexander von Humboldt International Conference on Climate Change, Natural Hazards, and Societies. Merida, Yucatán, Mexico 15-19 marzo 2010. Disponible en: URL: http://meetings.copernicus.org/avh6

33. Monroy C, Bustamante DM, Pineda SS, Rodas A, Castro X, Ayala V, Quiñónes, et al. House improvements and community participation in the control of Triatoma dimidiata re-infestation in Jutiapa, Guatemala. Cad. Saúde Pública. 2009;25(suppl 1)168-78.

34. Patz JA, Graczyk TK, Geller N, Vittor AY. Effects of environmental Change on emerging parasitic diseases. Int. J. Parasitol. 2000 Nov;30(12-13):1395405.

35. Bundy D, Guyatt H. Schools of health: focus on health, education and the school-age child. Parasitol Today. 1996 Aug;12(8):1-1 